\title{
Multipole expansions for energy and momenta carried by gravitational waves
}

\author{
Milton Ruiz • Miguel Alcubierre • Darío Núñez • \\ Ryoji Takahashi
}

\section{Erratum to: Gen Relativ Gravit (2008) 40:1705-1729 DOI 10.1007/s10714-007-0570-8}

Due to a typesetting error, the title was incorrect and should read as above.

The online version of the original article can be found under doi:10.1007/s10714-007-0570-8.

M. Ruiz $(\varangle) \cdot$ M. Alcubierre · D. Núñez $\cdot$ R. Takahashi

Instituto de Ciencias Nucleares, Universidad Nacional Autónoma de México, A.P. 70-543, Mexico DF 04510, Mexico

e-mail: ruizm@nucleares.unam.mx

M. Alcubierre

e-mail: malcubi@nucleares.unam.mx

R. Takahashi

e-mail: ryoji@nucleares.unam.mx

Present Address:

D. Núñez

Max-Planck-Institut für Gravitationsphysik, Albert Einstein Institut, 14476 Golm, Germany

e-mail:nunez@nucleares.unam.mx 